\title{
ON DUALITY OF PROBABILITIES FOR CARD DEALING
}

\author{
JEFFREY S. ROSENTHAL
}

(Communicated by Lawrence Gray)

\begin{abstract}
We simplify and generalize a result of Sobel and Frankowski concerning the equality in distribution of certain random quantities associated with dealing cards
\end{abstract}

Sobel and Frankowski [1] recently described a notion of "duality" for carddealing probabilities. They prove the equality of certain probabilities for different card-dealing schemes, and then discuss applications of their work. Their proof is quite computationally involved. The purpose of this short note is to present a much simpler and more conceptual proof of their result, which also generalizes it substantially.

Consider a deck of $N$ cards, called Deck \#1, which contains $b$ different "categories" (e.g., suits), of sizes $m_{1}, \ldots, m_{b}$. Consider dealing (uniformly at random) $j$ different hands from this deck, of sizes $n_{1}, \ldots, n_{j}$. Without loss of generality we assume that

$$
m_{1}+\cdots+m_{b}=n_{1}+\cdots+n_{j}=N
$$

(since if not, we could always include an extra category or an extra hand to make this so). For clarity, the case $N=52, b=j=4, m_{1}=\cdots=m_{4}=$ $n_{1}=\cdots=n_{4}=13$ corresponds to dealing an ordinary bridge game.

For $1 \leq x \leq b$ and $1 \leq y \leq j$, we let $C_{x y}$ by the random variable representing the number of cards from category $x$ dealt to hand $y$. Let $C$ record these numbers as a random $b \times j$ matrix.

To explain the duality notion, consider a second deck of $N$ cards, Deck \#2, which has the sizes of categories and hands reversed. Thus, this second deck has $j$ different categories of sizes $n_{1}, \ldots, n_{j}$, and we deal out $b$ different hands of sizes $m_{1}, \ldots, m_{b}$. We let $D_{x y}$ be the random variable representing the number of cards from category $x$ in hand $y$. Let $D$ record these numbers as a random $j \times b$ matrix.

Received by the editors December 15, 1992 and, in revised form, March 31, 1993.

1991 Mathematics Subject Classification. Primary 60C05. 
Theorem. The matrix $D$ has the same probability distribution as does the transpose of the matrix $C$. In symbols, $D \stackrel{d}{=} C^{\mathrm{t}}$.

Remark. In [1], only the case

$$
m_{1}=\cdots=m_{b-1} \text { and } n_{1}=\cdots=n_{j-1}
$$

is considered. It is proved there that

$$
P\left(\max _{\substack{1 \leq x \leq b-1 \\ 1 \leq y \leq j-1}} C_{x y} \leq r\right)=P\left(\max _{\substack{1 \leq x \leq j-1 \\ 1 \leq y \leq b-1}} D_{x y} \leq r\right)
$$

for any $r$ (and similarly with $\leq$ replaced by $\geq$ ). Our theorem thus generalizes [1] in two ways. First, we allow unequal category and hand sizes. Second, we prove the equality of the full joint distributions, not just the equality of specific probabilities.

Example. For example, in the case of an ordinary bridge game, the theorem says that the probability distribution of the four suits in any given hand is equal to the probability distribution of spades among the four hands. In particular, it shows [1] that the probability that a given hand has no more than four cards of any one suit is the same as the probability that none of the hands contains more than four spades.

Proof of the theorem. We describe here a simple proof of this theorem. Our proof is based on a coupled construction for dealing the two decks simultaneously, under which the matrices $D$ and $C^{\mathrm{t}}$ are actually equal. (This is a standard method of showing equality in distribution.)

Let Deck \#1 and Deck \#2 be as above. Deal the cards from Deck \#1 uniformly at random (in any order), producing $j$ hands of sizes $n_{1}, \ldots, n_{j}$.

Deal the cards from Deck \#2 simultaneously, as follows: Each time a card of category $x$ is dealt to hand $y$ in Deck \#1, deal a card from category $y$ to hand $x$ in Deck \#2.

When Deck \#1 is completely deal out, Deck \#2 will also be completely dealt out, into $b$ hands of sizes $m_{1}, \ldots, m_{b}$. Also, it is easily checked that Deck \#2 will have been dealt out with the "proper probabilities", i.e., with the cards dealt out uniformly at random. Thus, we have constructed a coupling for dealing out the two decks.

However, it is easily seen that for $1 \leq x \leq j$ and $1 \leq y \leq b$,

(\# cards of category $x$ in hand $y$ for Deck \#1) = (\# cards of category $y$ in hand $x$ for Deck \#2).

This shows that for this coupling, we have $D=C^{\mathfrak{t}}$. The result about distributions follows.

\section{ACKNOWLEDGMENTS}

I am grateful to Milton Sobel and Krzysztof Frankowski for sharing their unpublished work with me. I thank Bert Fristedt and Naresh Jain for advice and encouragement. 


\section{REFERENCES}

1. M. Sobel and K. Frankowski, $A$ duality theorem for solving multiple-player mutlivariate hypergeometric problems, Technical Report, Department of Computer Science, University of Minnesota, 1992.

School of Mathematics, University of Minnesota, Minneapolis, Minnesota 55455

Current address: Department of Statistics, University of Toronto, Toronto, Ontario, Canada M5S 1A1

E-mail address: jeff@utstat.toronto.edu 